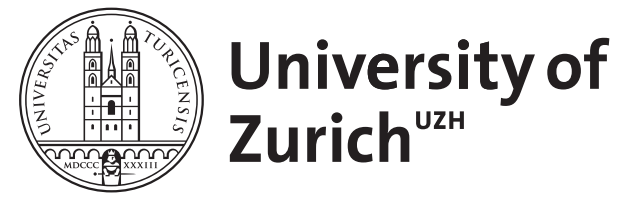

\title{
Schwierige Kindesentwicklung bei der Sectio
}

\author{
Zimmermann, Roland
}

\begin{abstract}
Ein tief im Becken eingeklemmter fetaler Kopf (,,impacted head“) beim Kaiserschnitt ist der Albtraum jedes Geburtshelfers. Ohne ein gutes Konzept geht die Entwicklung mit einer hohen kindlichen und mütterlichen Morbidität einher. Die von uns bevorzugte Strategie in einer solchen Situation ist die Entwicklung aus umgekehrter Steißlage, weil dabei ausschließlich Zugkräfte ausgeübt werden. Die sonst praktizierte Push-und-Pull-Methode wird nur angewendet, wenn das erste Verfahren versagt, weil Druck am fetalen Kopf zu Frakturen und Hirnblutungen führen kann. Aufgrund eigener Erfahrungen und einer umfassenden Literaturrecherche werden alle bekannten Manöver im Detail beschrieben. = A deeply impacted fetal head during cesarean section is a nightmare for obstetricians. Without a clear concept of how to disengage the head, the delivery leads to high fetal and maternal morbidity. In this situation we prefer the reverse breech extraction strategy because it exclusively requires pulling forces. The widely used pull and push method is only used as a second line manoeuver as pushing the fetal head can lead to skull fractures and intracranial hemorrhage. The article is based on experiences of a single center and on a comprehensive literature search. All known manoeuvers for delivering a baby safely are described in detail.
\end{abstract}

DOI: https://doi.org/10.1007/s00129-015-3752-6

Posted at the Zurich Open Repository and Archive, University of Zurich ZORA URL: https://doi.org/10.5167/uzh-207570

Journal Article

Published Version

Originally published at:

Zimmermann, Roland (2015). Schwierige Kindesentwicklung bei der Sectio. Der Gynäkologe, 48(8):599610.

DOI: https://doi.org/10.1007/s00129-015-3752-6 


\section{Redaktion}

T. Dimpfl, Kassel

W. Janni, Ulm

R. Kreienberg, Landshut

N. Maass, Kiel

O. Ortmann, Regensburg

B. Sonntag, Hamburg

K. Vetter, Berlin

R. Zimmermann, Zürich

\section{Punkte sammeln auf ... springermedizin.de/ eAkademie \\ Teilnahmemöglichkeiten \\ Diese Fortbildungseinheit steht Ihnen als e.CME und e.Tutorial in der Springer Medizin e.Akademie zur Verfügung. \\ - e.CME: kostenfreie Teilnahme im Rahmen des jeweiligen Zeitschriften- abonnements \\ - e.Tutorial: Teilnahme im Rahmen des e.Med-Abonnements}

\section{Zertifizierung}

Diese Fortbildungseinheit ist mit $3 \mathrm{CME}-$ Punkten zertifiziert von der Landesärztekammer Hessen und der Nordrheinischen Akademie für Ärztliche Fort- und Weiterbildung und damit auch für andere Ärztekammern anerkennungsfähig.

\section{Hinweis für Leser aus Österreich} Gemäß dem Diplom-Fortbildungs-Programm (DFP) der Österreichischen Ärztekammer werden die in der e.Akademie erworbenen CME-Punkte hierfür 1:1 als fachspezifische Fortbildung anerkannt.

\section{Kontakt und weitere Informationen}

Springer-Verlag GmbH

Springer Medizin Kundenservice

Tel. 08007780777

E-Mail:kundenservice@springermedizin.de

\section{CME Zertifizierte Fortbildung}

\section{R. Zimmermann}

Klinik für Geburtshilfe, UniversitätsSpital Zürich, Zürich, Schweiz

\section{Schwierige Kindesentwicklung bei der Sectio}

\section{Zusammenfassung}

Ein tief im Becken eingeklemmter fetaler Kopf (,impacted head“) beim Kaiserschnitt ist der Albtraum jedes Geburtshelfers. Ohne ein gutes Konzept geht die Entwicklung mit einer hohen kindlichen und mütterlichen Morbidität einher. Die von uns bevorzugte Strategie in einer solchen Situation ist die Entwicklung aus umgekehrter Steißlage, weil dabei ausschließlich Zugkräfte ausgeübt werden. Die sonst praktizierte Push-und-Pull-Methode wird nur angewendet, wenn das erste Verfahren versagt, weil Druck am fetalen Kopf zu Frakturen und Hirnblutungen führen kann. Aufgrund eigener Erfahrungen und einer umfassenden Literaturrecherche werden alle bekannten Manöver im Detail beschrieben.

\section{Schlüsselwörter}

Dystokie · Geburtswehen · Entbindung · Steißlage · Hämorrhagie 


\section{Lernziele}

\section{Nach Lektüre dieses Beitrages \\ - ist Ihnen bekannt, dass die Sectiorate in den meisten entwickelten Ländern mittlerweile auf über $\mathbf{3 0} \%$ angestiegen ist und die höchste Anstiegsrate bei der sekundären Sectio zu beobachten ist. \\ - wissen Sie, dass eine Sectio bei vollständig eröffnetem Muttermund für Mutter und Kind ein bisweilen deutlich erhöhtes Risiko birgt. Denn dabei ist die Uterotomie- Entbindungszeit massiv verlängert, das Kind erleidet unter Umständen Verletzungen und die Geburt hinterlässt bei der Mutter erhebliche Rissverletzungen mit erhöhtem Blutverlust. \\ - sind Sie sicher, dass auch solche schwierigen Situationen erfolgreich gemeistert werden können, wenn man einige Tricks bei der Operation berücksichtigt.}

\section{Einleitung}

Dieser Beitrag soll dem Leser helfen, sich auf eine Sectio mit erhöhtem Risiko vorzubereiten und die Entwicklung des Kindes situationsgerecht und unter Zuhilfenahme entsprechender Hilfsmittel richtig auszuführen mit dem Ziel, Schäden bei Kind und Mutter zu minimieren.

Verschiedene Faktoren haben in den letzten 25 Jahren in allen westlichen Ländern zu einer

Die Anstiegsrate ist am höchsten bei den sekundären Sectiones

Für das Kind steigt das Risiko einer Hypoxie durch die verlängerte Uterotomie-Entbindungszeit ansteigenden Sectiorate geführt. Am meisten zugenommen hat die Rate an sekundären Sectiones, d. h. derjenigen, die erst unter der Geburt indiziert und durchgeführt werden [1]. Beunruhigend ist dabei der Anstieg der Sectiones, die bei vollständig eröffnetem Muttermund durchgeführt werden [2]. Diese Sectiones können technisch extrem schwierig sein, weil der Kopf häufig im kleinen Becken wie ein Sektkorken festklemmt und kaum in Richtung Uterotomie zu luxieren ist (• Abb. 1). Sie sind deshalb mit einem erheblichen Gesundheitsrisiko für Mutter und Kind verbunden [3]. Für die Mutter steigt die Wahrscheinlichkeit, dass die Uterotomie T-förmig oder auch im Bogen nach kranial erweitert werden muss, dass zusätzliche Risse in Richtung von Parametrien oder Vagina auftreten mit einem erheblichen Blutverlust und erhöhter Transfusionswahrscheinlichkeit. Für das Kind steigt das Risiko einer Hypoxie durch die verlängerte Uterotomie-Entbindungszeit. Schädelfrakturen, intrakranielle Blutungen, aber auch Knochenbrüche an den Extremitäten sind gehäuft beschrieben. Der Verzicht auf eine schwierige vaginal-operative Entbindung mit erhöhter fetomaternaler Morbidität wird also erkauft durch einen Anstieg der sekundären Sectio mit gleichwohl erhöhter Morbidität. Durch den Druck, den ersten Kaiserschnitt zu verhindern [4], indem die akzeptierte Dauer der aktiven Austreibungsperiode auf mindestens 3 Stunden bei Erstgebärenden heraufgesetzt wird, wird die Häufigkeit von schwierigen Sectiones in Zukunft noch weiter ansteigen.

\section{Difficult delivery of babies during cesarean section}

\begin{abstract}
A deeply impacted fetal head during cesarean section is a nightmare for obstetricians. Without a clear concept of how to disengage the head, the delivery leads to high fetal and maternal morbidity. In this situation we prefer the reverse breech extraction strategy because it exclusively requires pulling forces. The widely used pull and push method is only used as a second line manoeuver as pushing the fetal head can lead to skull fractures and intracranial hemorrhage. The article is based on experiences of a single center and on a comprehensive literature search. All known manoeuvers for delivering a baby safely are described in detail.
\end{abstract}

\section{Keywords}

Dystocia $\cdot$ Obstetric labor $\cdot$ Childbirth $\cdot$ Breech presentation $\cdot$ Hemorrhage 


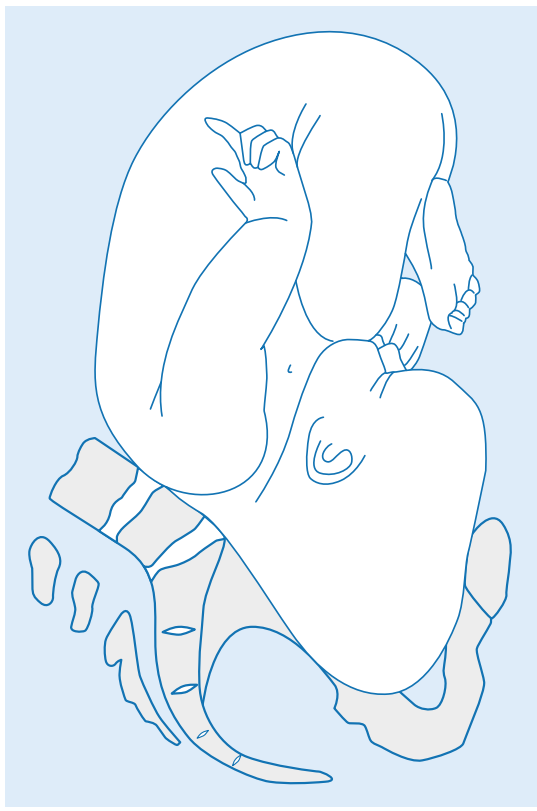

Abb. 1 \ Bei dorsoposteriorem „hohem" Gradstand ist gelegentlich der Kopf wie ein Sektkorken ins kleine Becken hineingepresst, was ein Herausluxieren bei der Sectio erschwert
Es ist deshalb von entscheidender Bedeutung, die Schäden durch die technisch sehr anspruchsvollen Sectiones mithilfe einer Reihe von Maßnahmen zu mindern. In diesem Beitrag möchte ich auf die beiden häufigsten Probleme bei einer Sectio eingehen:

- den tief im Becken impaktierten Kopf einerseits und

- den hoch im Kavum flottierenden Kopf andererseits.

Die westliche Literatur schweigt sich erstaunlicherweise $\mathrm{zu}$ diesem Thema weitgehend aus. In den letzten 50 Jahren sind in Europa und den USA nur spärlich Artikel zu diesem Thema erschienen. Die meisten Arbeiten zu diesem Thema stammen aus Asien oder Afrika (Indien, Iran, Äthiopien, Nigeria etc.). Dortige Kollegen haben wertvolle Techniken beschrieben und ausgewertet. Nicht eingehen werde ich auf andere schwierige Situationen, wie die Entwicklung des Kindes aus Querlage, bei Placenta praevia, Placenta increta, einer im Myometrium und Anhydramnion eingemauerten Frühgeburt oder der Entwicklung bei großem Myom im Uterotomiebereich.

\section{Sectio bei tief im kleinen Becken impaktiertem Kopf (zephale Dystokie)}

Analog zur Schulterdystokie haben wir anlässlich einer Sectio die Situation, dass der fetale Kopf wie ein Sektkorken tief im kleinen Becken steckt und mit keiner üblichen Maßnahme herausgezogen werden kann, ,zephale Dystokie“ genannt (• Abb. 1). Wer je in einer solchen Situation war, weiß, dass der Vergleich absolut berechtigt ist. Was der Albtraum Schulterdystokie bei der vaginalen Geburt ist, ist die zephale Dystokie bei der Sectio. Es beginnt schon bei der Eröffnung des Uterus. Entweder liegt die Uterotomie direkt über dem fetalen Mund oder Hals oder es quillt gleich der vorne liegende Arm hervor. Es folgen verzweifelte Versuche, mit der Hand zwischen den Augen des Feten und der Symphyse unter den Kopf des Kindes zu kommen, um ihn in üblicher Weise aus dem Becken zu luxieren. Zug an den kindlichen Schultern nach oben zur Luxation des Kopfes ist fast obligat von einer starken Kontraktion des Uterus begleitet, welche den Kopf noch tiefer ins Becken drückt. Zudem entsteht beim Zug nach oben in der Vagina ein Vakuum, welches dem Zug entgegen wirkt. Nach vielen Minuten, die fast wie eine Ewigkeit erscheinen, gelingt es schließlich, mit Druck von vaginal den Kopf zu mobilisieren und das Kind zu entwickeln. Das schlaff-weiße Kind muss der Neonatologie zur primären Reanimation übergeben werden, während man sich den stark blutenden Rissen in die Parametrien widmet, die bei der traumatischen Kindsentwicklung entstanden sind. Bluttransfusionen und febrile Heilungsverläufe sind oft Zusatzkomplikationen bei der Mutter.

\section{Risikofaktoren}

Eine zephale Dystokie kommt nahezu ausschließlich vor bei einer Sectio unter der Geburt. Verschiedene Risikofaktoren begünstigen diese Komplikation, gut beschriebene Faktoren sind in - Tab. 1 zusammengefasst.

\section{Komplikationen einer zephalen Dystokie}

Unkontrollierte Extraktionsversuche können sowohl beim Kind als auch bei der Mutter gravierende Schäden verursachen [5, 6, 7]. Gut dokumentiert sind beim Kind Schädelfrakturen oder intrakranielle Hämatome durch zu großen Druck auf den Kopf (• Abb. 2). Aber auch Plexusparesen oder Arm- und Klavikulafrakturen sind beschrieben. Durch die oftmals stark verlängerte

Entscheidend ist es, Schäden durch technisch sehr anspruchsvolle Sectiones zu mindern

In der westlichen Literatur der letzten $\mathbf{5 0}$ Jahre gibt es kaum Publikationen zum Thema

Zug an den kindlichen Schultern nach oben zur Luxation des Kopfes ist fast obligat von einer starken Uteruskontraktion begleitet

Bluttransfusionen und febrile Heilungsverläufe sind oft maternale Zusatzkomplikationen 
Eine zephale Dystokie bei der Sectio ist das Pendant zur Schulterdystokie bei der vaginalen Geburt

Von großer Bedeutung ist die palpatorische oder sonographische exakte Bestimmung der Einstellung

Das Eröffnen des Uterus in der Austreibungsperiode ist fast obligat von einer starken Dauerkontraktion begleitet

I.d.R. tritt ein fetaler Kopf im Querdurchmesser ins Becken ein und rotiert beim Tiefertreten um $90^{\circ}$ in den geraden Durchmesser
Uterotomie-Entwicklungszeit sind die Kinder deprimiert und azidotisch.

Eine zephale Dystokie bei der Sectio ist das Pendant zur Schulterdystokie bei der vaginalen Geburt. Der Kopf ist tief im kleinen Becken eingeklemmt und lässt sich mit der üblichen Technik bei der Sectio nicht mobilisieren. Fehlerhafte Extraktionsmanöver können zu erheblichen Problemen bei Mutter und Kind führen. Mit einer adaptierten Technik können Kollateralschäden für beide minimiert werden.

\section{Prozedere im Detail}

\section{Vorbereitung}

Eine erfolgreiche Entbindung bei zephaler Dystokie beginnt bei der Vorbereitung. Ist trotz vollständig eröffnetem Muttermund die Leitstelle für eine vaginal-operative Entbindung zu hoch und die Indikation zur Sectio gestellt, sollte der Eingriff mit erhöhter Dringlichkeit, begonnen werden, d. h. in maximal 20-40 min. Von großer Bedeutung ist auch die palpatorische oder sonographische exakte Bestimmung der Einstellung, insbesondere die Stellung des fetalen Rückens. Es lohnt sich, die Wehen bis zum Operationseginn mit einer $\beta$-Mimetika-Tokolyse i. v. zu reduzieren.

Die Lagerung auf dem Operationstisch erfolgt mit etwa $40^{\circ}$ gegrätschten Beinen, um im Bedarfsfall einer Assistenzperson Zugang zur Vagina zu ermöglichen. Hilfsmaterialien (• Abb. 3) müssen für den Bedarfsfall bereit stehen. Da mit einer intensiven Betreuung des Kindes zu rechnen ist, sollte der Neonatologe oder zumindest ein für das Kind zuständiger Arzt, der nicht in die Operation integriert ist, dazu gerufen werden.

Unmittelbar vor Desinfektion der Haut soll nochmals durch einen erfahrenen Arzt vaginal untersucht werden, ob nicht in der Zwischenzeit die Geburt vaginal-operativ zu Ende gebracht werden kann [8].

\section{Operationsbeginn und Relaxation des Uterus}

Bei eine Sectio in der Austreibungsperiode ist das untere Uterinsegment häufig stark ausgezogen. Bei einem Eingehen auf der gewohnten Höhe trifft man nicht selten auf die stark nach kranial gezogene Vagina statt auf den Uterus. Aus diesem Grund soll bereits der Pfannenstiel-Hautschnitt etwa $2 \mathrm{~cm}$ weiter kranial als üblich erfolgen. Die Uterotomie erfolgt zusätzlich etwa $4 \mathrm{~cm}$ höher als üblich. Durch dieses Vorgehen schafft man für die Entwicklung mehr Platz und verhindert ein Eröffnen der Vagina. Zusätzlich kann im Fall von Schwierigkeiten durch seitliches Erweitern der Laparotomie mehr Platz geschaffen werden.

Das Eröffnen des Uterus in der Austreibungsperiode ist fast obligat von einer starken Dauerkontraktion begleitet. Üblicherweise dauern Kontraktionen 30-40 s. Dieses Intervall muss man verstreichen lassen, bis man an eine Entwicklung denken kann. Man kann die Zeit aber nutzen, um zu explorieren, ob man mit seiner Hand zwischen Kopf und Symphyse gelangen kann, um den Kopf in üblicher Weise aus dem Becken zu luxieren. Im Falle einer Uterus(dauer)kontraktion verwenden wir eine Notfalltokolyse mit $\operatorname{dem} \beta$-2-Sympathomimetikum Hexoprenalin (Gynipral ${ }^{\oplus}$ ) $5 \mu \mathrm{g}(=1 \mathrm{ml})$ verdünnt mit $9 \mathrm{ml} \mathrm{NaCl} \mathrm{0,9 \%} \mathrm{i.} \mathrm{v.,} \mathrm{verabreicht} \mathrm{in} \mathrm{Dosen} \mathrm{von} \mathrm{1-2} \mathrm{ml}$ im Abstand von $60 \mathrm{~s}$ [9]. Alternativen sind Fenoterol (Partusisten ${ }^{\circledR}$ ) oder Nitroglycerin $50 \mu \mathrm{g}$ i. v. mit maximal 4 weiteren Dosen im Abstand von je 60 s [10]. Sollte eine Dauerkontraktion mit diesen Maßnahmen nicht behebbar sein, kommt als Ultima Ratio nur eine sofortige Intubation mit tiefer Narkose in Frage.

Im Folgenden gehe ich davon aus, dass es trotz guter Uterusrelaxation und ggf. Handwechsel nicht gelingt, die Hand zwischen Kopf und Symphyse unter den Kopf zu bringen und dass auch der Versuch, mit Druck von der Seite den Kopf zu rotieren, nicht erfolgreich ist.

\section{Berücksichtigung des Geburtsmechanismus}

Im Regelfall tritt der fetale Kopf im queren Durchmesser ins mütterliche Becken ein und rotiert beim Tiefertreten wie ein Schlüssel im Schlüsselloch um $90^{\circ}$ in den geraden Durchmesser. Wenn 

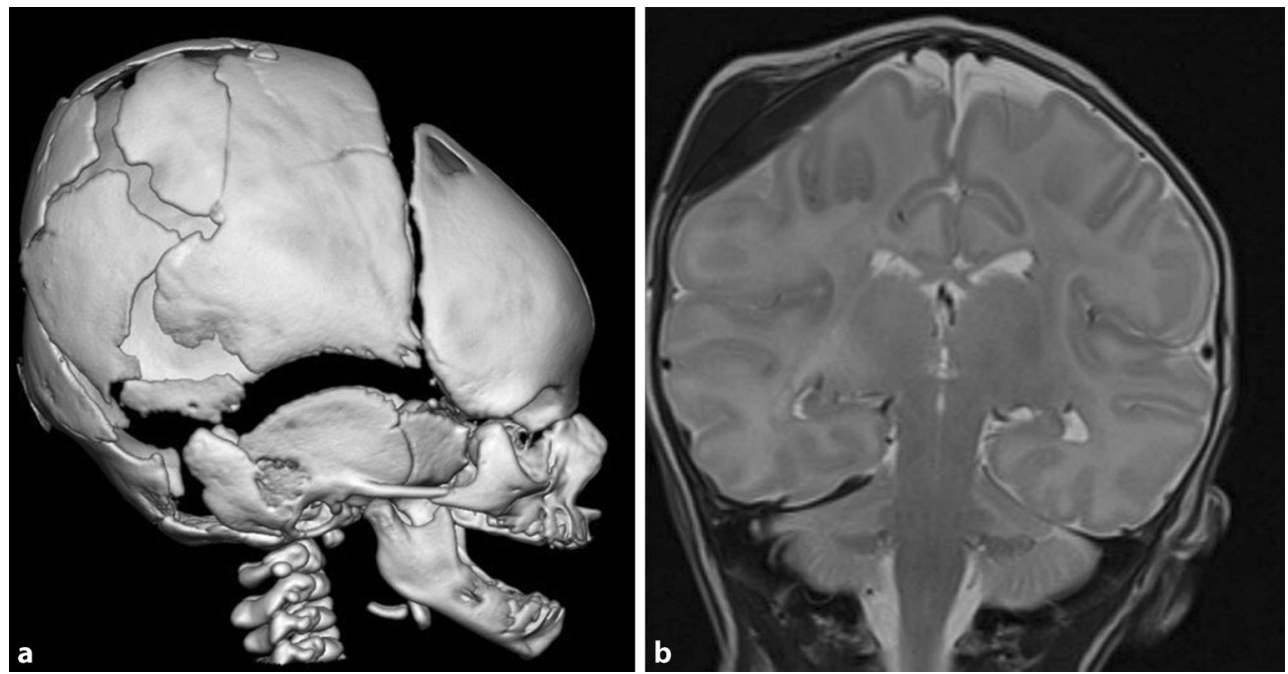

Abb. $2 \Delta$ a, b Schädelfraktur (a) und intrakranielles Hämatom (b) nach Finger-Push-Entwicklung

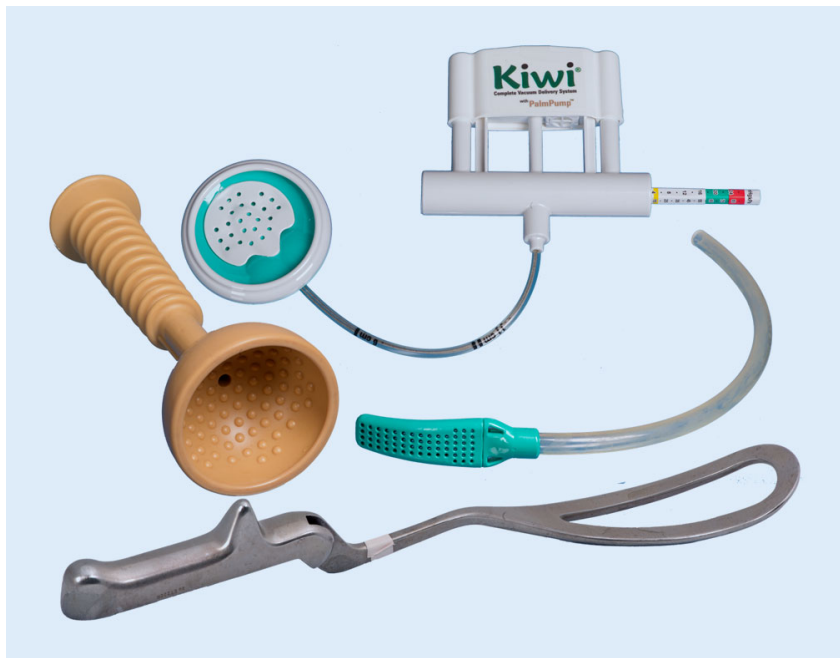

Abb. $3<$ Hilfsmittel: Mehrweg Silc Cup 60mm (mit freundlicher Genehmigung der Medela AG), Kiwi ${ }^{\circledR}$ Omni-C, Halbzange, C-Snorkel (mit freundlicher Genehmigung von Clinical Innovations LLC)

bei einer sekundären Sectio der Kopf wieder aus dem Becken heraus soll, muss der Vorgang in einer ersten Phase exakt rückwärts ablaufen. Ist dies gelungen, folgt in der zweiten Phase das Hervorluxieren aus der Uterotomie. Dazu ein Bild: Ein Knopf kann auf nur eine einzige Weise durch ein Knopfloch gedrückt werden: Er muss parallel zum Knopfloch ausgerichtet sein und man muss ihn um $90^{\circ}$ abkippen, damit er stromlinienförmig durch das Knopfloch passt. Analog muss bei Entwickeln eines Kopfes aus der Uterotomie dieser erst in den queren Durchmesser rotiert und ggf. flektiert werden, damit der Flexionspunkt [11] in die Mitte der Uterotomie gelangt. Das Hervorhebeln entspricht dann dem Abkippen des Knopfes.

Steckt der Kopf tief im Becken fest, gelingt es bei ausreichender uteriner Relaxation in einfacheren Fällen, durch schrägen Zug an der vorderen fetalen Schulter nach oben das Kind aus dem Becken herauszuschrauben.

Gelingt diese einfache Methode nicht, ist das beste weitere Vorgehen abhängig von der Stellung des fetalen Rückens.

\section{Vorgehen bei dorsoposteriorem Gradstand}

Im Folgenden wird die Entwicklung aus umgekehrter Steißlage beschrieben. Diese geht wohl am ehesten auf den indischen Arzt Patwardhan zurück [12]. Bereits 1957 beschrieb er in einer Übersichtsarbeit zur Sectio die Möglichkeit, im Fall einer zephalen Dystokie das Kind von den Füßen her als umgekehrte Steißlagengeburt zu entwickeln. In den mehr als 50 Jahren sind

Bei zephaler Dystokie kann von den Füßen her als umgekehrte Steißlagengeburt entwickelt werden 

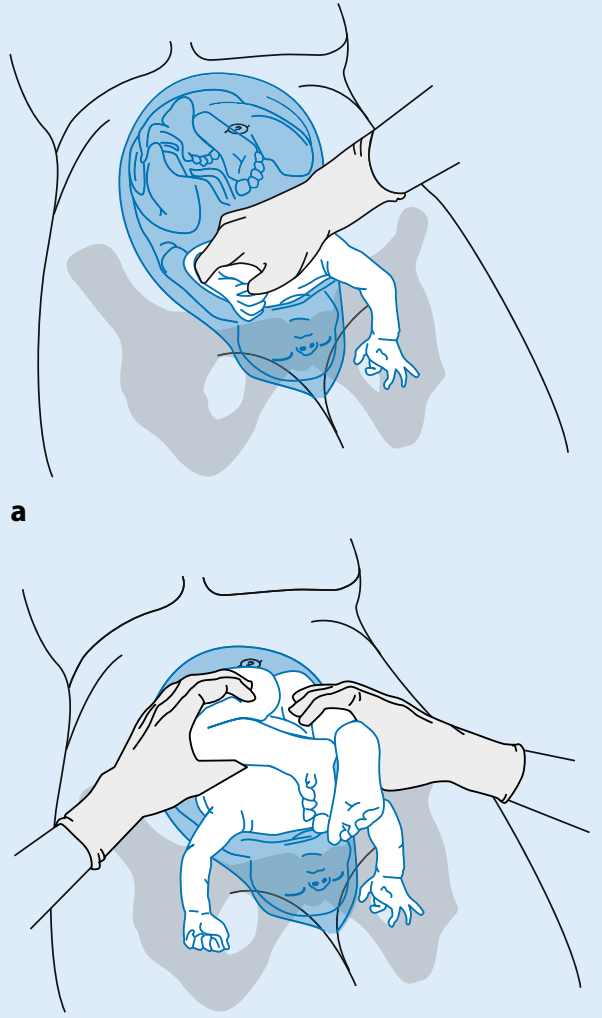

c

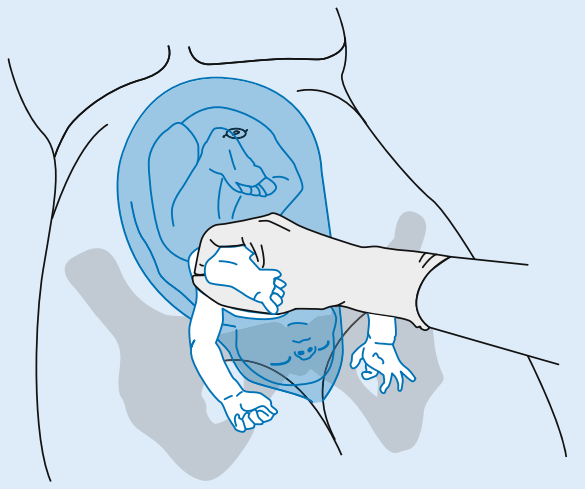

b

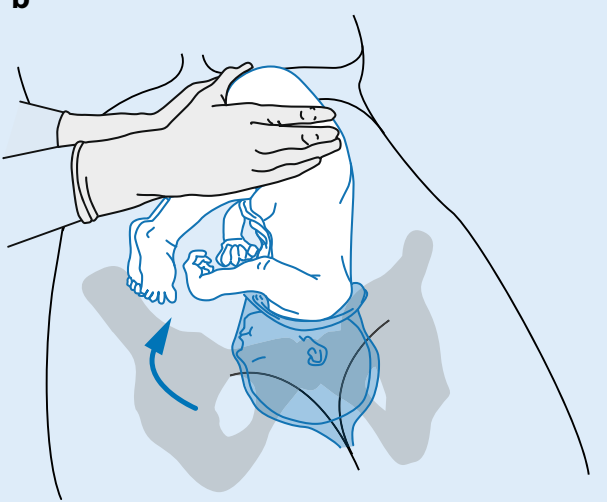

d

Abb. $4 \Delta$ a Kind in dorsoposteriorer Lage, beide Arme entwickelt, b Griff an den Fuß mit Extraktion von Bein 1, cEntwicklung des Körpers durch Zug an beiden Beinen, $\mathbf{d}$ Entwicklung des Kopfes durch Schraubbewegung an den Schultern

Bei der Entwicklung aus umgekehrter Steißlage sind seltener Erweiterungen der Uterotomie erforderlich

vorzugsweise in Asien und Afrika ein gutes Dutzend Fallserien und Fall-Kontroll-Studien zu diesem Thema publiziert worden.

Die äthiopischen Ärzte Berhan und Berhan haben kürzlich die Erfahrung dieser Arbeiten zusammengefasst [13]. Verglichen mit der unten beschriebenen Push-und-Pull-Methode gelingt mit dieser Technik die Entwicklung des Kindes schneller und schonender, deutlich seltener sind Erweiterungen der Uterotomie notwendig. Gestützt auf die Literatur und auf zahlreiche eigene erfolgreiche Fälle an unserer Klinik gehen wir im Detail wie folgt vor (• Abb.4a-d).

In einem ersten Schritt werden beide Arme sorgfältig aus dem Uterus herausgefädelt und vor die mütterliche Bauchdecke gebracht. Dies verhindert eine nach der Extraktion von Beinen und Rumpf später notwendige sehr schwierige Armlösung. Als zweiter Schritt werden die fetalen Füße aufgesucht. Dies kann durchaus zunächst nur einen Fuß betreffen. Kranialer Zug der oberen Uterotomiehälfte durch den Assistenten kann bei knappen Verhältnissen hilfreich sein. Bei sehr schwierigen Platzverhältnissen ist selten eine Erweiterung des Uterotomie - T- oder bogenförmig - notwendig. Reicht auch das noch nicht aus, ist eine Erweiterung der Laparotomie in Erwägung zu ziehen. Als dritter Schritt werden durch bestimmten und kontinuierlichen Zug an beiden Oberschenkeln der fetale Steiß und dann der Rumpf extrahiert. Ein leichter Druck auf den Fundus durch den Assistenten kann dabei hilfreich sein. Beim letzten Schritt wird das Kind mit dem Bracht-Handgriff gefasst und der Kopf wird mit einer Drehbewegung (wie oben beschrieben) aus dem Grad- in den Querstand gedreht. Durch Druck von außen durch den Assistenten rutscht dann der Kopf mühelos durch die Uterotomie. 


\section{Vorgehen bei dorsoanteriorem Gradstand}

Liegt der fetale Rücken vorne, kommt man mit der oben beschriebenen Methode nicht an die Füße, und da der Kopf im kleinen Becken fixiert ist, kann der Fet auch nicht in seiner Achse in eine dorsoposteriore Stellung rotiert werden.

Für diesen Fall hat ebenfalls Patwardhan eine Lösung präsentiert (• Abb. 5). Die Publikation mit der größten Fallzahl zu dieser Technik stammt von Mukhopadhyay [14]. Bei dieser Methode werden zunächst der vordere Arm bzw. die vordere Schulter, danach durch leichte Rotation des Kindes der hintere Arm bzw. Schulter aus der Uterotomie entwickelt und durch sanften symmetrischen Zug an den Armen vor die mütterliche Bauchdecke gebracht. Danach geht man mit dem Zeig- und Mittelfinger an beiden Seiten des Kindes ein und extrahiert das Kind durch Zug seitlich am Stamm bzw. in den Hüften. Der Zug am Kind muss durch fundalen Druck durch den Assistenten unterstützt werden. Der letzte Schritt, die Entwicklung des Kopfes, verläuft dann wieder wie oben beschrieben.

\section{Push-und-Pull-Methode}

Landesman und Graber haben vor 30 Jahren die vielerorts praktizierte Methode „Push und Pull“ beschrieben [15]. Bei dieser Methode wird der Rotationszug an den fetalen Schultern unterstützt durch eine vollständig in die Vagina eingebrachte Hand, die den Kopf nach oben drückt (• Abb. 6).

In der Originalarbeit empfiehlt Landesman die primäre Lagerung der Patientin in Steinschnittlage, vornehmlich auf Beinstützen. Der Assistent, der den Kopf hochschiebt, steht für dieses Manöver zwischen den Beinen.

Die Möglichkeit, ohne großen Zeitverlust auf eine Push-und-Pull-Methode zu wechseln, ist der Grund, aus dem wir bei allen sekundären Sectiones die Frauen mit gegrätschten Beinen lagern. Dies erleichtert den Zugang für eine Hilfsperson im Bedarfsfall wesentlich.

Da es in solchen Fällen sehr schwierig ist, wie bei einer manuellen Plazentalösung die ganze Hand in die Vagina einzuführen, wird häufig nur mit den Fingern gedrückt, was die Gefahr einer fetalen Schädelfraktur sowie von Hirnblutungen beinhaltet.

Als Alternative zur Hand bzw. den Fingern verwenden wir zum Drücken des Kopfes den $60 \mathrm{~mm}$ Silc Cup Silicone Vacuum von Medela/Menox. Ursprünglich wurde er dazu hergestellt, mit einem Vakuum am fetalen Kopf angebracht zu werden, doch wir verwenden ihn hier als Schub-, nicht als Zuginstrument. Durch die Silikonkappe wird beim Hinaufdrücken der Druck wesentlich besser auf die Schädelkalotte verteilt, als wenn man mit den Fingern hochschiebt. Zudem ist der Stiel deutlich länger als menschliche Finger. Analog zur Vakuumextraktion muss sich die Stoßrichtung an der Führungslinie durch das Becken halten, d. h. bei einer kindlichen Leitstelle auf interspinal steil nach oben.

Die Frage, welche Methode schneller und mit weniger Schäden für Mutter und Kind zum Ziel führt, die Entwicklung aus umgekehrter Steißlage oder die Push-und-Pull-Methode, kann zum heutigen Zeitpunkt nicht abschließend beantwortet, da bislang keine randomisierte Studie vorliegt, die beide Vorgehensweisen verglichen hätte.

Aufgrund der Literatur [13] und der sehr positiven eigenen Erfahrungen mit der Entwicklung aus umgekehrter Steißlage steht diese Methode bei uns an oberster Stelle. Diese Haltung wird auch dadurch gestützt, dass der menschliche Körper besser auf Zug als auf Druck belastet werden kann. Trotzdem ist es wichtig, auch die beschriebene Alternative im geburtshilflichen Repertoire zu haben, da immer ein Fall auftreten kann, bei dem die primär gewählte Methode nicht zum Ziel geführt hat.

\section{Lösen eines Vakuums als zusätzliche Hilfsmethode}

Beim Versuch, einen tief im Becken eingeklemmten Kopf herauszuluxieren, entsteht um die Zervix und Vagina ein Vakuum, das den Kopf sofort wieder ins Becken zieht. Zur Lösung dieses Vakuums hat die Firma Clinical Innovations ein schnorchelartiges Rohr mit weicher Silikonspitze entwickelt, den C-Snorkel ${ }^{\circledR}$. Er wird gemäß Herstellerangaben vor Operationsbeginn mit der grünen Spitze dorsal zwischen Kopf und Zervix eingelegt und während des Eingriffs dort belassen. Barrier et al. beschreiben in einer retrospektiven Fall-Kontroll-Studie ein erleichterte Entwicklung des Kindes bei impaktiertem Kopf [16]. Wir haben selber mit dem C-Snorkel ${ }^{\circledR}$ nur begrenzt Erfahrung, da die meisten Fälle durch eine umgekehrte Steißextraktion problemlos entwickelt werden können.
Liegt der Rücken vorn und ist der Kopf im kleinen Becken fixiert, kann der Fet nicht in seiner Achse in eine dorsoposteriore Stellung rotiert werden

Der Rotationszug an den fetalen Schultern wird unterstützt, indem die Hand den Kopf nach oben drückt

Analog zur Vakuumextraktion muss sich die Stoßrichtung an der Führungslinie durch das Becken halten

Beide Alternativen sind wichtig für das geburtshilfliche Repertoire

Beim Luxationsversuch des im Becken eingeklemmten Kopfes entsteht ein Vakuum, das den Kopf sofort wieder zurückzieht 


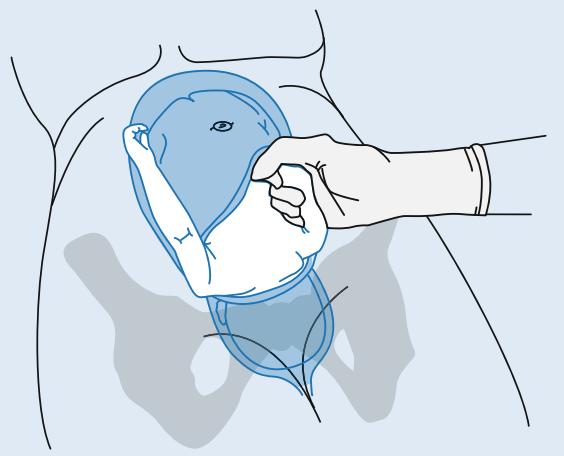

a

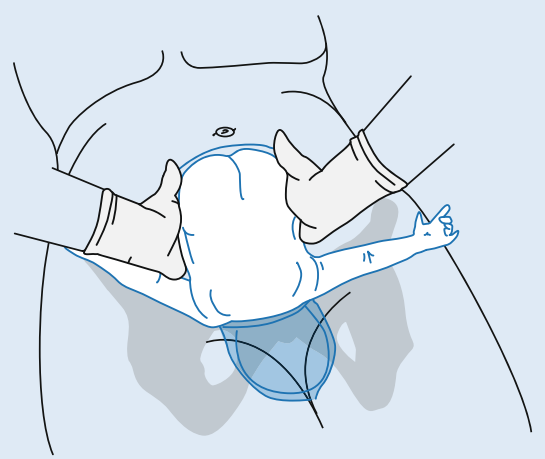

c

Abb. $5 \Delta$ a Kind in dorsoanteriorer Lage, beide Arme entwickelt, b Griff an Stamm/Hüfte mit beiden Händen, c Entwicklung des Körpers durch Zug an den Hüften mit Druck auf den Fundus, d Entwicklung des Kopfes durch Schraubbewegung an den Schultern

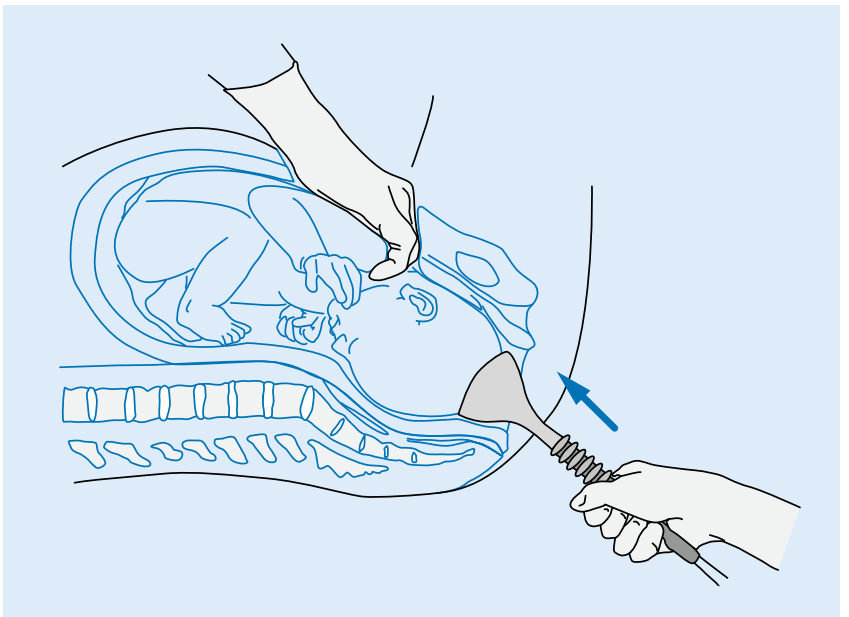

Abb. $6<$ Pull-Push-Methode, Silc Cup vaginal eingeführt am Kopf, Schub auf den Kopf und Pull an den Schultern

Das Prinzip klingt jedoch logisch, was uns dazu bewogen hat, für sekundäre Sectiones einen CSnorke ${ }^{\circledR}$ griffbereit zu haben, um ihn nötigenfalls während der Operation einzulegen.

\section{Gefährliche Methoden}

Bei einer geplanten Sectio mit anthropoidem Becken der Mutter ist die Verwendung eines einzelnen Zangenblattes (z. B. halbe Geburtszange, Murless Extractor, Coyne Spoon) anstatt der 
etwas dickeren Hand eine elegante Hilfe, den Kopf aus dem Becken heraus zu luxieren. Bei impaktiertem Kopf dagegen sind solche Instrumente gefährlich, denn sie erhöhen das Risiko von Verletzungen von Mutter und Kind. Da mit den oben beschriebenen Methoden Kinder praktisch immer erfolgreich entwickelt werden können, haben solche Hilfsmittel keinen Platz. Ähnlich verhält es sich mit der Empfehlung, in solchen Fällen ein Sectio-Vakuum durchzuführen. Da der Kopf tief im Becken eingekeilt ist, kann eine Pelotte gar nicht über dem Flexionspunkt angesetzt werden, was die Erfolgsquote drastisch reduziert und die Komplikationsrate erhöht [17, 18].

\section{Revision der mütterlichen Weichteile}

Ist das Kind erst einmal erfolgreich entwickelt, muss eine sorgfältige Revision der mütterlichen Weichteile durchgeführt werden. Im Zusammenhang mit derart erschwerten Entwicklungen sind massive Weichteilverletzungen zu befürchten. Das untere Uterinsegment ist durch die oftmals lange Geburt dünn ausgezogen und rissig. Die Folge sind Rissverletzungen seitlich in die Parametrien, die sich manchmal unter dem viszeralen Peritoneum verstecken, oder Risse in die Vagina. Zur besseren Übersicht über die Weichteile ist es nützlich, den Uterus vor die mütterliche Bauchdecke nach kranial zu ziehen. Notwendige T-förmige Erweiterungen der Uterotomie sollten mit wenigen weitgreifenden Nähten versorgt werden, um die Ischämie im Wundgebiet gering zu halten. Seit einer eitrig-nekrotischen Uterotomie nach einer solchen Sectio verwenden wir nur noch antimikrobiell beschichtetes Vicryl ${ }^{\circledR}$ als Nahtmaterial. Eine einmalige Antibiotikaprophylaxe vor Beginn der Sectio ist sowieso Standard.

Sectiones mit erschwerter Entwicklung sind mit einem erhöhten Weichteilschadenspotenzial am Uterus verbunden. Um Nachblutungen und Infekten vorzubeugen ist eine sorgfältige Revision notwendig.

\section{Sectio bei flottierendem Kopf}

Ganz anders als das Problem eines tief im Becken impaktierten Kopfes ist ein hoch im Uterus flottierender Kopf. Der Kopf wird durch den Bandl-Kontraktionsring zurückgehalten, was einem korrekten Ausrichten des Kopfes zur Uterotomie entgegenwirkt. Speziell bei viel Fruchtwasser kann es enorm schwierig werden, den Kopf in die Öffnung zu dirigieren.

In solchen Situationen sind 2 Methoden sicher und zielführend:

- die Verwendung einer Vakuumglocke,

- alternativ die innere Wendung auf den Steiß mit anschließender Extraktion.

Für das Vakuum verwenden wir die KIWI ${ }^{\circledR}$ Omni-C Glocke, welche speziell für die Anwendung im Zusammenhang mit einer Sectio konzipiert wurde. Die Glocke ist etwas schmaler als ihr vaginaloperativer Bruder, hat Fingerfurchen zur besseren Platzierung und im inneren der Glocke kein Schaumkissen, das akzidentell herausfallen und im mütterlichen Abdomen verbleiben könnte. Da sie ohne Strom auskommt und als „single-use item“ steril verpackt geliefert wird, eignet sie sich hervorragend für diese Situation. Wie bei allen Vakuumentbindungen ist es entscheidend, die Glocke über dem Flexionspunkt anzubringen, was ein genaues Tasten der Schädelnähte und Fontanellen erfordert. Wenn man die Rille für den Zugdraht parallel zur Sagittalnaht ausrichtet, bekommt man zudem eine Idee, wie bei Zug der Kopf dreht. Die Publikationen zu dieser Methode sind zwar spärlich [19, 20,21], im Gegensatz zum Einsatz des Vakuums bei impaktiertem Kopf sind jedoch keine gravierenden Komplikationen bei Kind und Mutter beschrieben und die Methode führt schneller zum gewünschten Erfolg als eine manuelle Extraktion.

Falls das Platzieren einer Vakuumglocke nicht gelingt, wird die Alternative eingesetzt: die Wendung auf den Steiß. Dies erfordert einerseits etwas Kraft zur Überwindung des muskulären Widerstandes beim Einführen der Hand in den Uterus, andererseits Geschick im Auffinden des ersten Fußes. Sobald dieser einwandfrei identifiziert ist, kann ähnlich wie bei Querlagen durch Zug am Bein und gleichzeitigem Hochschieben des Kopfes die innere Wendung eingeleitet werden. Bei hohem uterinen Tonus kann auch eine Notfalltokolyse (s. oben) nützlich sein.

Können schwierige Entwicklungen bei Sectio geübt werden?

Verschiedene Katastrophen und Rechtsfälle im Zusammenhang mit sehr schwierigen Entwicklungen bei der Sectio haben zumindest in Großbritannien zur Einsicht geführt, dass eine gezielte Instruktion und ein Training sehr wichtig wären [22].
Bei impaktiertem Kopf sind manche geburtshilflichen Instrumente gefährlich, sie erhöhen das Verletzungsrisiko

Durch die oft lange Geburt ist das untere Uterinsegment dünn ausgezogen und rissig

V. a. bei viel Fruchtwasser kann es schwierig werden, den Kopf in die Öffnung zu dirigieren

Wie bei allen Vakuumentbindungen ist es entscheidend, die Glocke über dem Flexionspunkt anzubringen

Misslingt das Platzieren einer Vakuumglocke, wird die Wendung auf den Steiß eingesetzt

Auch unter forensischen Aspekten ist ein gezieltes Training im Zusammenhang mit schwierigen Entwicklungen bei der Sectio wichtig 
Die Firma Adam, Rouilly (Vertrieb in Deutschland durch Schultes medacta), hat unter Anleitung eines britischen Teams einen Simulator entwickelt, der speziell die Entwicklung bei impaktiertem Kopf üben lassen soll. Er ist unter dem Namen „Desperate Debra“ bekannt. Auf der Website von Scientific American findet sich ein Clip zum Simulator [23]. Die Entwickler des Simulators haben anhand von 3 typischen Situationen die Nützlichkeit für Ärzte in Weiterbildung analysiert [24]. Fast 90 \% beurteilten die Handhabung als sehr realitätsnah, und über $90 \%$ stuften den Simulator als nützlich für das Training ein. An der eigenen Klinik haben wir den Simulator seit 2013 in Betrieb. Bestätigen können wir, dass die Situation eines tief im kleinen Becken impaktierten Kopfes sehr gut zur Geltung kommt. Da das Modell fast ausschließlich Druck am Kopf (Flexion, Rotation, Druck nach oben) erlaubt und keinen Zug an den (fehlenden) Schultern, kann auch die Push-und-Pull-Methode nur teilweise geübt werden. Die Entwicklung aus umgekehrter Steißlage ist gar nicht möglich. Trotzdem hat dieser nicht sehr kostspielige Simulator in Kursen einen festen Platz, weil er sehr realitätsnah die Situation, die unverhofft bei einer Sectio auftreten kann, vermittelt.

\section{Fazit für die Praxis}

- Das Problem eines bei der Sectio tief im kleinen Becken impaktierten Kopfes, wir nennen es in Anlehnung an die Schulterdystokie „zephale Dystokie“, ist einer der Albträume jedes geburtshilflichen Operateurs, weil es mit einer erheblichen Morbidität von Mutter und Kind einhergeht.

- Mit einer korrekten Operationsvorbereitung, einer adäquaten Relaxation des Uterus bei der Entwicklung und einem klaren Plan können jedoch die meisten Komplikationen vermieden werden.

- Die wichtigste Methode ist die Entwicklung aus umgekehrter Steißlage, alternativ kommt die Push-und-Pull-Methode zur Anwendung. Da der menschliche Körper besser auf Zug als auf Druck gebaut ist, bevorzugen wir die umgekehrte Steißlagenextraktion.

- Ein systematisches Training in der Weiterbildung ist dringend nötig.

\section{Korrespondenzadresse}

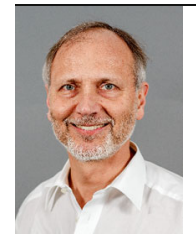

Prof. Dr. R. Zimmermann

Klinik für Geburtshilfe, UniversitätsSpital Zürich

Frauenklinikstr. 10, 8091 Zürich, Schweiz

roland.zimmermann@usz.ch

\section{Einhaltung ethischer Richtlinien}

Interessenkonflikt. R. Zimmermann gibt an, dass kein Interessenkonflikt besteht.

Dieser Beitrag beinhaltet keine Studien an Menschen oder Tieren.

\section{Literatur}

1. Loudon JAZ, Groom KM, Hinkson L, Harrington D, Paterson-Brown $S$ (2010) Changing trends in operative delivery performed at full dilatation over a 10-year period. J Obstet Gynaecol 30:370-375

2. Unterscheider J, McMenamin M, Cullinane $F$ (2011) Rising rates of caesarean deliveries at full cervical dilatation: concerning trend. Eur J Obstet Gynaecol Reprod Biol 157:141-144
3. Sung JF, Daniels KI, Brodzinsky L, El-Sayed YY, Caughey AB, Lyel DJ (2007) Cesarean delivery outcomes after a prolonged second stage of labor. Am J Obstet Gynecol 197:306.e1

4. ACOG/SMFM (2014) Obstetric Care Consensus No. 1: safe prevention of the primary cesarean delivery. Obstet Gynecol 123:693-711

5. Murphy DJ, Liebling RE, Verity L, Swingler R, Patel R (2001) Early maternal and neonatal morbidity associated with operative delivery in second stage of labour: a cohort study. Lancet 358:1203-1207

6. Häger RME, Daltveit AK, Hofoss D, Nilsen ST, Kolaas T, Oian P,Henriksen $\mathrm{T}$ (2004) Complications of cesarean deliveries: rates and risk factors. Am JObstet Gynecol 190:428-434

7. Laughon SK, Verghella V, Reddy UM, Sundaram R, Lu Z, Hoffman MK (2014) Neonatal and maternal outcome with prolonged second 
stage of labor. Obstet Gynecol 124:57-67

8. Olah KS (2005) Reversal of the decision for caesarean section in the second stage of labour on the basis of consultant vaginal assessment. J Obstet Gynaecol 25:115-116

9. Zimmermann R (2012) Handbuch Geburtshilfe. Eigenverlag, Zürich

10. David $M$, Halle $H$, Lichtenegger W, Sinha P, Zimmermann T (1998) Nitroglycerin to facilitate fetal extraction during cesarean delivery. Obstet Gynecol 91:119-124

11. Zimmermann R (2012) Vakuumentbindung - richtig ausgeführt Gynäkologe 10:791-800

12. Patwardhan BD, Motashaw ND (1957) Cesarean section. J Obstet Gynecol India 8:1-15

13. Berhan Y, Berhan A (2014) A meta-analysis of reverse breech extraction to deliver a deeply impacted head during cesarean delivery. Int J Gynecol Obstet 124:99-105

14. Mukhopadhyay $P$, Naskar T, Dalui R (2005) Evaluation of Patwardhan's technic - a four year study in a rural teaching hospital. J Obstet Gynecol India 55:244-246

15. Landesman R, Graber EA (1984) Abdominovaginal delivery: modification of the cesarean section operation to facilitate delivery of the impacted head. Am J Obstet Gynecol 148:707-710

16. Barrier BF, Allison JL, Andelin CO, Drobnis EZ (2013) A simple device prevents hysterotomy extensions during cesarean delivery for failed second stage of labor. Gynecol Obstet Invest 76:90-94

17. Clark SL, Vines VL, Belfort MA (2008) Fetal injury associated with routine vacuum use during cesarean delivery. Am J Obstet Gynecol 198:e4

18. Fareeduddin R, Schifrin BS (2008) Subgaleal hemorrhage after the use of a vacuum extractor during elective cesarean delivery: a case report. JReprod Med 53:809

19. Pelosi MA, Apuzzio J (1984) Use of the soft silicone obstetric vacuum cup for delivery of the fetal head at cesarean section. J Reprod Med 29:289-292

20. Dimitrov A, Pavlova E, Krusteva K, Nikolov A (2008) Caesarean section with vacuum extraction of the head. Akusherstvo Ginekol 47:3-6

21. Sritippayawan S, Chantrapitak W (2011) Assisted delivery of high floating fetal head: a comparison of vacuum-assisted delivery with manual extraction. Asian Biomed 5:699-703

22. Sethuram R, Jamjute $P$, Kevelighan E (2010) Delivery of the deeply engaged head: a lacuna in training J Obstet Gynaecol 30:545-549
23. www.scientificamerican.com/ video.cfm?id=desperate-debrahelps-doctors-pract2012-08-17. Zugegriffen am: 13.11.2012

24. Vousden N, Hamakarim Z, Briley A, Girling J, Seed PT, Tydeman G Shennan AH (2015) Assessment of a full dilation cesarean delivery simulator. Obstet Gynecol 125:369-374 


\section{CME-Fragebogen}

Bitte beachten Sie:

- Teilnahme nur online unter: springermedizin.de/eAkademie

- Die Frage-Antwort-Kombinationen werden online individuell zusammengestellt.

- Es ist immer nur eine Antwort möglich.

? Welche der folgenden Methoden ist bei einer Sectio am wenigsten geeignet zur schnellen Relaxation des Uterus?

$\square 40$ s abwarten

$\square$ Nitroglycerin i. v.

Bolus eines $\beta$-Mimetikums i. v.

Nifedipinkapsel $10 \mathrm{mg}$ sublingual

Tiefe Narkose der Mutter

Was ist die letzte Maßnahme vor Operationsbeginn einer Sectio in der Austreibungsperiode?

$\square$ Neonatologie avisieren

$\square$ Lagerung mit gegrätschten Beinen

$\square$ Nochmalige vaginale Untersuchung

$\square$ Bereitstellen einer Vakuumglocke

$\square$ Bereitstellen eines Tokolytikums

Was ist die typische Einstellung bei einer zephalen Dystokie?

$\square$ Hoher Gradstand

$\square$ Tiefer Querstand

$\square$ Vorderer Asynklitismus

$\square$ Hinterer Asynklitismus

$\square$ Hoher Schultergradstand

(2) Was begünstigt das Auftreten einer zephalen Dystokie nicht?

$\square$ Ein hoher Gradstand

$\square$ Die Deflexion des kindlichen Kopfes

$\square$ Ein Vakuum zwischen dem kindlichen Kopf und den Geburtswegen

$\square$ Eine Lumbalisation von S1

$\square$ Ein langes Intervall zwischen Indikationsstellung zur Sectio und Operationsbeginn
Wo liegt der Punkt für die richtige Positionierung der Vakuumglocke bei einer Sectio?

$\square$ Unabhängig von der Einstellung immer über dem Flexionspunkt

$\square$ Über der kleinen Fontanelle

$\square$ Über der großen Fontanelle

$3 \mathrm{~cm}$ posterior der kleinen Fontanelle

An keiner der o. g. Stellen

Welche Methode ist bei zephaler Dystokie ungünstig?

Ein Herausschrauben des Kindes durch schrägen Zug an der vorderen Schulter

Die Entwicklung aus umgekehrter Steißlage

Das Heraufhebeln des fetalen Kopfes aus dem kleinen Becken mittels eines Zangenlöffels

Das Hinaufdrücken des fetalen Kopfes von vaginal

Das Relaxieren des Uterus

Welcher ist der erste Schritt bei der Extraktion aus umgekehrter Steißlage?

$\square$ Identifizierung eines Fußes

$\square$ Zug am einen Bein

Erweiterung der Uterotomie

Starker Druck von fundal

Entwicklung beider Arme

Bei der Push-Methode

ist die Stoßrichtung unabhängig von der Führungslinie.

zeigt die Schubrichtung horizontal.

spielt der Höhenstand der Leitstelle keine Rolle. spielt eine starke Deflexion des Kopfes keine Rolle.

$\square$ muss der Druck auf den Schädel verteilt werden.

Was gehört nicht zur Sectio in der Austreibungsphase?

$\square$ Eine leicht höher gelegte PfannenstielLaparotomie

$\square$ Ein hohes Eingehen bei der Uterotomie

$\square$ Das Hervorziehen des Uterus vor die Bauchdecke nach Geburt des Kindes

$\square$ Die sorgfältige Revision der Uterotomie

$\square$ Das Einlegen eines Bakri ${ }^{\mathrm{TM}}{ }^{-B a l l o n s}$ zur Atonieprophylaxe

Welche ist die wirksamste Maßnahme, wenn bei einer Sectio der Kopf beim Versuch inn herauszuhebeln immer abrutscht?

$\square$ Die T-förmige Erweiterung der Uterotomie

Die Tonisierung des Uterus durch Oxytocin

$\square$ Das Ablassen von Fruchtwasser

$\square$ Die Durchführung einer Vakuumextraktion

$\square$ Die Durchführung einer Zangenextraktion

Diese zertifizierte Fortbildung ist 12 Monate auf springermedizin.de/ eAkademie verfügbar.

Dort erfahren Sie auch den genauen Teilnahmeschluss. Nach Ablauf des Zertifizierungszeitraums können Sie diese Fortbildung und den Fragebogen weitere 24 Monate nutzen. 